medRxiv preprint doi: https://doi.org/10.1101/2020.09.15.20195081; this version posted September 18, 2020. The copyright holder has placed this preprint (which was not certified by peer review) in the Public Domain. It is no longer restricted by copyright. Anyone can legally share, reuse, remix, or adapt this material for any purpose without crediting the original authors.

\title{
An Application of Logistic Formula to Deaths by COVID-19 in Japan
}

\author{
Takesi Saito* \\ Department of Physics, Kwansei Gakuin University, Sanda 669-1337, Japan
}

\begin{abstract}
A logistic formula in biology is applied to analyze deaths by COVID-19 for both of the first and the second waves in Japan. We then discuss ends of both waves and their mortality ratios. The meaning of population $N$ in an epidemic is discussed.
\end{abstract}

\section{Introduction}

The SIR model [1] in the theory of infection is powerful to analyze an epidemic about how it spreads and how it ends [2-8]. The SIR model is composed of three equations for $S, I$ and $R$, where they are numbers for susceptibles, infectives and removed, respectively. Three equations can be solved numerically, if two parameters $\alpha$ and $c$ are given, where $\alpha$ is the basic reproduction number and $c$ the removed ratio.

In Sec. 2 we summarize approximate solutions of the SIR equations, based on the logistic formula in biology [9], which have been derived in a previous work [10]. These approximate solutions have simple forms, so that they are very useful to discuss an epidemic. In Sec. 3 these solutions are applied to fix the basic reproduction number $\alpha$ and the removed ratio $c$, especially from data of accumulated number of deaths by COVID-19 for the first wave in Japan. Here, our policy is to use only data of deaths, but not of cases. The mortality ratio for the first wave is also calculated. In addition we discuss the meaning of population $N$ in an epidemic. In Sec. 4 same things are given for the second wave. The final section is devoted to concluding remarks. In Appendix data of deaths for the first and the second waves are summarized.

\section{A logistic curve from the SIR model}

Equations of the SIR model [1] are given by

$$
\begin{aligned}
& \frac{d S(t)}{d t}=-b I(t) S(t), \\
& \frac{d I(t)}{d t}=(b S(t)-c) I(t), \\
& \frac{d R(t)}{d t}=c I(t),
\end{aligned}
$$

*tsaito@k7.dion.ne.jp

NOTE: This preprint reports new research that has not been certified by peer review and should not be used to guide clinical practice. 
medRxiv preprint doi: https://doi.org/10.1101/2020.09.15.20195081; this version posted September 18, 2020. The copyright holder has placed this preprint (which was not certified by peer review) in the Public Domain. It is no longer restricted by copyright. Anyone can legally share, reuse, remix, or adapt this material for any purpose without crediting the original authors.

where $S, I$ and $R$ are numbers for susceptibles, infectives and removed, respectively, $b$ the infection ratio and $c$ the removed ratio. From Eqs. (2.1) and (2.3) we get $d S / d R=-\alpha S$, $(\alpha=b / c)$, which is integrated to be

$$
S=\exp (-\alpha R)
$$

where $\alpha$ stands for the basic reproduction number. In the same way, from Eqs. (2.2) and (2.3), we have $d I / d R=\alpha S-1=\alpha \exp (-\alpha R)-1$, which is integrated to be

$$
I(R)=1-R-\exp (-\alpha R) .
$$

The solutions (2.4) and (2.5) satisfy boundary conditions $S=1$ and $I=0$ at $R=0$. We normalize the total number to be unity, i.e., $S+I+R=1$. Since $I(t)$ is 0 when $t \rightarrow \infty$, we have $1-R(\infty)-\exp [-\alpha R(\infty)]=0$ from Eq.(2.5). Hence, it follows a useful formula

$$
\alpha=-\frac{\ln [1-R(\infty)]}{R(\infty)} .
$$

Some exact formulas at the infection peak $t=T$ are summarized as follows:

$$
\begin{aligned}
& R(T)=\frac{\ln \alpha}{\alpha}, \\
& I(T)=1-\frac{1}{\alpha}-\frac{\ln \alpha}{\alpha} .
\end{aligned}
$$

The first one is derived as follows: Since the peak point is given by $d I / d t=c(\alpha S-1) I=0$ at $t=T$, we get $S(T)=\exp [-\alpha R(T)]=1 / \alpha$. This proves Eq.(2.7). The second equation (2.8) directly follows by $I(T)=1-R(T)-S(T)=1-\ln \alpha / \alpha-1 / \alpha$.

Let us summarize logistic formulas for SIRs functions [10]. The third equation (2. 3) can be written as

$$
\frac{d R}{d t^{\prime}}=I\left(t^{\prime}\right)=1-R-\exp (-\alpha R), \quad t^{\prime}=c t,
$$

with $t$ the true time. Let us expand the exponential factor in the second order of $x=\alpha R$,

$$
\exp (-x) \approx 1-x+\frac{x^{2}}{2}
$$

Then we have

$$
\frac{d R}{d t^{\prime}}=1-R-\left(1-\alpha R+\frac{\alpha^{2} R^{2}}{2}\right)=A(B-R) R
$$

with $A=\alpha^{2} / 2, B=2(\alpha-1) / \alpha^{2}$. This equation is a type of the logistic growth curve in the biology [9], easily solved as

$$
R(t)=\frac{R(\infty)}{1+\exp (-z)}, \quad z=a c(t-T)
$$

where $A B=\alpha-1=a$ and $B=R(\infty)=2(\alpha-1) / \alpha^{2}$. Inserting $R(t)$ into $I(t)=(1 / c) d R / d t$, we have

$$
I(t)=\frac{a R(\infty)}{2(1+\cosh (z))}, \quad a=\alpha-1 .
$$

Note that peak values of $I(t)$ and $R(t)$ at $t=T$ are given by

$$
I(T)=\frac{a R(\infty)}{4} \quad \text { and } \quad R(T)=\frac{R(\infty)}{2} .
$$

Hence we have a useful formula

$$
\frac{I(T)}{R(T)}=\frac{a}{2}
$$


medRxiv preprint doi: https://doi.org/10.1101/2020.09.15.20195081; this version posted September 18, 2020. The copyright holder has placed

\section{Application to COVID-19 for the first wave}

We make use of data [11] of deaths rather than cases. It is our policy not to use data of cases. Define the mortality ratio $\lambda$ by

$$
\lambda=\frac{D(t)}{R(t)}
$$

where $D(t)$ is the accumulated number of deaths. For the population $N$, the total number of deaths at $t$ is given by

$$
N D(t)=\lambda N R(t)=\frac{d}{1+\exp (-z)}, \quad z=a c(t-T),
$$

where $d=N D(\infty)=\lambda N R(\infty)$ stands for the final total number of deaths. Note that from Eq.(3.2) we have a theorem, $N D(T)=d / 2$, which means the total number of deaths at the peak is just a half of the final ones.

Let us rewrite Eq.(3.2) into the form as

$$
-z=a c(T-t)=\ln F(t), \quad F=\frac{d}{N D(t)}-1 .
$$

Accordingly, for different times $t_{1}, t_{2}$ and $t_{3}$ we have

$$
\begin{aligned}
& a c\left(t_{2}-t_{1}\right)=\ln \left(\frac{F\left(t_{1}\right)}{F\left(t_{2}\right)}\right), \\
& a c\left(t_{3}-t_{2}\right)=\ln \left(\frac{F\left(t_{2}\right)}{F\left(t_{3}\right)}\right) .
\end{aligned}
$$

Table 1: Date and the total number of deaths in the first wave

\begin{tabular}{cc}
$\mathrm{t}$ & $\mathrm{N} \mathrm{D}(\mathrm{t})$ \\
\hline$t_{1}=95($ May 20) & 783 \\
$t_{2}=109($ June 3$)$ & 904.8 \\
$t_{3}=123($ June 17$)$ & 936
\end{tabular}

$(N D(t)$ is the total number of deaths in the first wave at $\mathrm{t}$ in Japan, which is an average of 5 -day deaths of $t-2, t-1, t, t+1, t+2$. The $t$ is the number of date starting from Feb. 16) A use is made of data of deaths [11]. Substituting data on the Table 1 into Eqs.(3.4) and (3.5), and eliminating $a c$, we get

$$
F\left(t_{1}\right) F\left(t_{3}\right)=F\left(t_{2}\right)^{2}
$$

This is nothing but the equation for $d$, that is,

$$
\left(\frac{d}{783}-1\right)\left(\frac{d}{936}-1\right)=\left(\frac{d}{904.8}-1\right)^{2} .
$$

We find a solution of this equation to be $\mathrm{d}=984$. According to the theorem $N D(T)=d / 2$, the number of deaths at $t=T$ is $d / 2=492$. This means the peak day is May 2nd, as seen on data. The result of $d=984$ is substituted into Eq.(3.4), then it follows

$$
a c=\frac{1}{14} \ln \left(\frac{F\left(t_{1}\right)}{F\left(t_{2}\right)}\right)=\frac{1}{14} \ln \left(\frac{0.26}{0.09}\right)=0.08
$$

In the following we take $c=0.04$, which is frequently quoted value. Then we have $a=$ $0.08 / 0.04=2.00$, that is, $\alpha=1+a=3$. Also, from the formula $(2.6)$ it follows $R(\infty)=0.94$. 
medRxiv preprint doi: https://doi.org/10.1101/2020.09.15.20195081; this version posted September 18, 2020. The copyright holder has placed this preprint (which was not certified by peer review) in the Public Domain. It is no longer restricted by copyright. Anyone can legally share, reuse, remix, or adapt this material for any purpose without crediting the original authors.

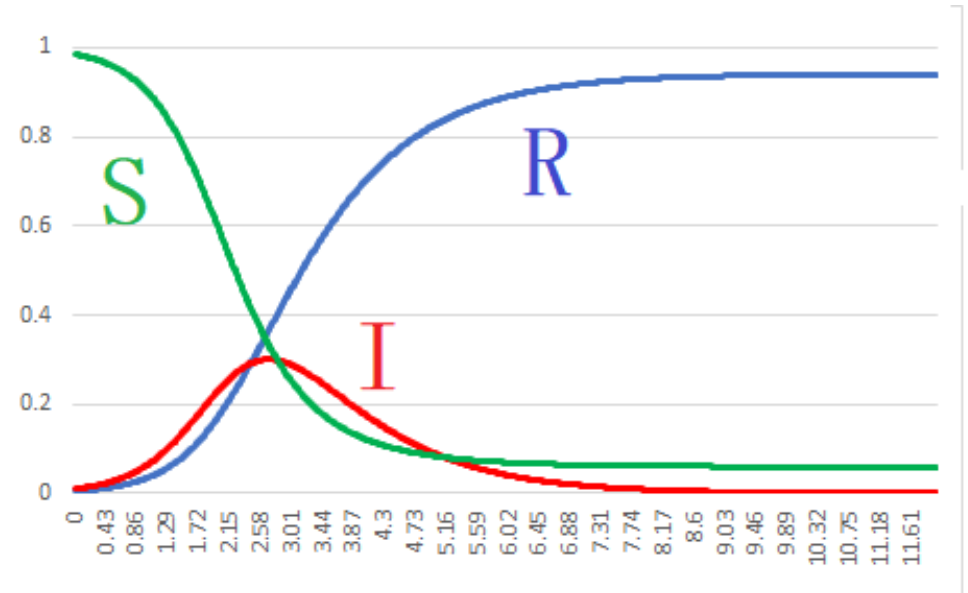

Figure 1: Graph of $S, I$ and $R$ for $\alpha=3$ and $c=0.04$

The original SIR equations are also solved by means of Excel. Their curves are drawn in Figure 1.

From the curve of $I\left(t^{\prime}\right)$ we find that $t^{\prime}=3.00$ gives a peak, which is compared with the formula $t^{\prime}=c t=0.04 \times 77=3.08$. This slightly differs from $t^{\prime}=3.00$. However, such a small difference may be allowed for a margin of error arising from our approximate method. After approximately 77 days later, the first epidemic will end around a middle of July(7/18) with the total number 984 of deaths.

Finally let us calculate the mortality ratio $\lambda=D(t) / R(t)$. The removed number $R(t)$ is given by $R(t)=r(t)+D(t)$, where $r(t)$ is the accumulated number of people discharged from hospital and $D(t)$ is the accumulated number of deaths. We find its mean value is $\langle\lambda\rangle=0.1$ with $\sigma=0.002$.

Here we have a comment about the population $N$. From Eq.(2.7) it follows $R(T)=\ln \alpha / \alpha=$ 0.37 for $\alpha=3$, whereas $N R(T)=4877$ from the data. Hence we get $N=4877 / R(T)=$ $4877 / 0.37 \approx 13000$. What is such a small population as $N=13000$ ? In order to discuss this question, we divide the Japan population into two groups A and B, A is immune to the present epidemic and B is not immune the epidemic. We can add also people who completely quarantine themselves to $\mathrm{A}$. We then conclude that such a small population as $N=13000$ corresponds to B, because $\mathrm{A}$ is irrelevant to infection.

\section{The second wave}

The second wave of COVID-19 in Japan seems to start from around June 22. The virus is now called the Tokyo type.

Table 2: Date and the number of deaths in the second wave

\begin{tabular}{cc}
$\mathrm{t}$ & $\mathrm{N} \mathrm{D}(\mathrm{t})$ \\
\hline$t_{1}=32$ (Aug. 20) & 171 \\
$t_{2}=38$ (Aug. 26) & 239.3 \\
$t_{3}=38$ (Sep. 1) & 309
\end{tabular}

$(N D(t)$ is the total number of deaths with 3 -day average in the second wave at $t$. The $t$ is 
medRxiv preprint doi: https://doi.org/10.1101/2020.09.15.20195081; this version posted September 18, 2020. The copyright holder has placed this preprint (which was not certified by peer review) in the Public Domain. It is no longer restricted by copyright. Anyone can legally share, reuse, remix, or adapt this material for any purpose without crediting the original authors.

the number of date starting from July 20. We make use of data in the appendix, where the number of deaths in the second wave is completely separated from that of the first wave. It is shown that the first death of the second wave appears on July 20.)

The corresponding equation to Eq.(3.7) is, from Table 2,

$$
\left(\frac{d}{171}-1\right)\left(\frac{d}{309}-1\right)=\left(\frac{d}{239.3}-1\right)^{2} .
$$

We have a solution $d=482$, so that $d / 2=241$ is the death number at the peak. Hence the peak day is Aug. 26 and $T=38$, as seen from Appendix.

The result of $d=482$ is substituted into Eq.(3.4), then it follows

$$
a c=\frac{1}{6} \ln \left(\frac{F\left(t_{1}\right)}{F\left(t_{2}\right)}\right)=0.1 .
$$

In the following we take $c=0.028$. Then we get $a=3.6$, that is, $\alpha=4.6$. Once having the basic reproduction number $\alpha=4.6$ with the removed ratio $c=0.028$, we can draw curves of $S, I$ and $R$ by means of Excel in Figure 2 .

From the curve of $I\left(t^{\prime}\right)$ we find that the peak day is $t^{\prime}=1.51$, which is compared with

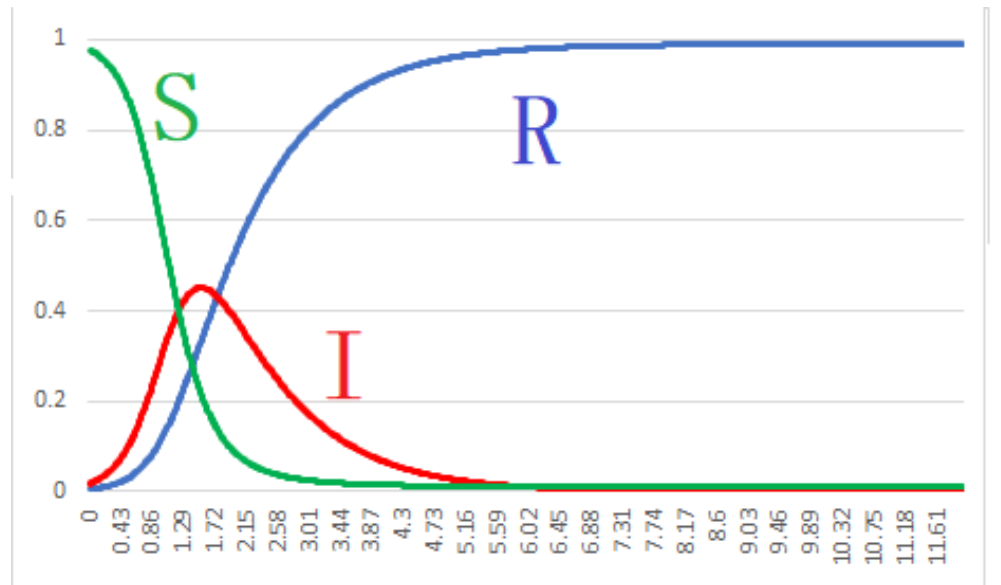

Figure 2: Graph of $S, I$ and $R$ for $\alpha=4.6$ and $c=0.028$

the formula $t^{\prime}=c t=0.028 \times 38=1.06$. This slightly differs from $t^{\prime}=1.51$. However, such a small difference may be allowed for a margin of error arising from our approximate method. Since the second infection begins from July 20, the $t^{\prime}=1.51$ corresponds with the true time $t=38$ days, so that the peak day is Aug. 26, with the total number 241 of deaths. After approximately 38 days later, the epidemic will end around Oct. 3 with the total number $241 \times 2=482$ of deaths. One can see that a ratio of both infectives on peak days is $I_{2} / I_{1}=3 / 2$, where $I_{1}$ and $I_{2}$ stand for infectives of $1^{\text {st }}$ and $2^{\text {nd }}$ waves.

Finally let us calculate the mortality ratio $\lambda=D(t) / R(t)$. We find that its mean value between July 20 and Aug. 22 is $\langle\lambda\rangle=0.005$ with $\sigma=0.0006$.

\section{Concluding remarks}

Our logistic formulas have been applied to analyze deaths by COVID-19 in Japan. Our policy is to use only data of deaths, but not of cases.

For the first wave, the basic reproduction number $\alpha$ is determined to be $\alpha=3$ and the removed 
medRxiv preprint doi: https://doi.org/10.1101/2020.09.15.20195081; this version posted September 18, 2020. The copyright holder has placed this preprint (which was not certified by peer review) in the Public Domain. It is no longer restricted by copyright. Anyone can legally share, reuse, remix, or adapt this material for any purpose without crediting the original authors.

ratio $c=0.04$. The corresponding curves of $S, I$ and $R$ are given in Figure 1 . From the curve of $I\left(t^{\prime}\right)$ we find that the infection peak day is May $2^{\text {nd }}$, then after approximately 77 days later, the first epidemic will end around a middle of July(7/18) with the total number 984 of deaths. The mortality ratio $\lambda=D(t) / R(t)$ is also calculated to be its mean value $\langle\lambda\rangle=0.1$ with $\sigma=0.002$.

Here we have a comment about the population $N$. We find $N$ being calculable from the formula $R(T)=\ln \alpha / \alpha=0.37$ for $\alpha=3$. Namely, from data we see $N R(T)=4877$ to give $N \approx 13000$. What is such a small population as $N=13000$ ? In order to discuss this question, we divide the Japan population into two groups $\mathrm{A}$ and $\mathrm{B}, \mathrm{A}$ is immune to the present epidemic and B is not immune the epidemic. We can add also people who completely quarantine themselves to $\mathrm{A}$. We then conclude that such a small population as $N=13000$ corresponds to $\mathrm{B}$, because $\mathrm{A}$ is irrelevant to infection.

For the second wave, the basic reproduction number $\alpha$ is determined to be $\alpha=4.6$ and removed ratio $c=0.028$. The corresponding curves of $S, I$ and $R$ are given in Figure 2. From the curve of $I\left(t^{\prime}\right)$ we find that the infection peak day is Aug. 26, then after approximately 38 days later, the second epidemic will end around Oct. 3, with the total number 482 of deaths. The mortality ratio $\lambda$ is also calculated to be its mean value $\langle\lambda\rangle=0.005$ with $\sigma=0.0006$. A ratio of both infectives of $1^{\text {st }}$ and $2^{\text {nd }}$ waves on peak days can be seen to be $2 / 3$.

\section{Acknowledgment}

The author would like to express his deep gratitude to K. Shigemoto for many valuable discussions and big supports. 


\section{Appendix:Data of accumulated number of deaths in the second wave}

$\begin{array}{cccccccc}\text { No. } & \text { month/day } & D_{12} & D_{2} & r_{12} & r_{2} & R_{2} & \lambda_{2} \\ 0 & 7 / 19 & 984 & 0 & 19523 & 0 & - & - \\ 1 & 7 / 20 & 987 & 3 & 19921 & 398 & 401 & 75 \\ 2 & 7 / 21 & 988 & 4 & 20403 & 880 & 884 & 45 \\ 3 & 7 / 22 & 989 & 5 & 20787 & 1264 & 1269 & 40 \\ 4 & 7 / 23 & 991 & 7 & 21080 & 1557 & 1564 & 44 \\ 5 & 7 / 24 & 992 & 8 & 21319 & 1796 & 1804 & 44 \\ 6 & 7 / 25 & 995 & 11 & 21514 & 1991 & 2002 & 55 \\ 7 & 7 / 26 & 995 & 11 & 21868 & 2345 & 2356 & 47 \\ 8 & 7 / 27 & 997 & 13 & 22560 & 3037 & 3050 & 42 \\ 9 & 7 / 28 & 1000 & 16 & 23256 & 3733 & 3749 & 43 \\ 10 & 7 / 29 & 1003 & 19 & 23928 & 4405 & 4424 & 43 \\ 11 & 7 / 30 & 1005 & 21 & 24678 & 5155 & 5176 & 41 \\ 12 & 7 / 31 & 1010 & 26 & 25253 & 5730 & 5756 & 45 \\ 13 & 8 / 1 & 1010 & 26 & 25653 & 6130 & 6156 & 42 \\ 14 & 8 / 2 & 1011 & 27 & 26234 & 6711 & 6738 & 40 \\ 15 & 8 / 3 & 1015 & 31 & 26944 & 7421 & 7452 & 42 \\ 16 & 8 / 4 & 1021 & 37 & 27775 & 8252 & 8289 & 45 \\ 17 & 8 / 5 & 1025 & 41 & 28624 & 9101 & 9142 & 45 \\ 18 & 8 / 6 & 1032 & 48 & 29900 & 10377 & 10425 & 46 \\ 19 & 8 / 7 & 1038 & 54 & 31054 & 11531 & 11585 & 47 \\ 20 & 8 / 8 & 1039 & 55 & 32059 & 12591 & 12591 & 44 \\ 21 & 8 / 9 & 1046 & 62 & 32805 & 13282 & 13344 & 46 \\ 22 & 8 / 10 & 1051 & 67 & 33722 & 14199 & 14266 & 46 \\ 23 & 8 / 11 & 1058 & 74 & 34635 & 15112 & 15186 & 49 \\ 24 & 8 / 12 & 1062 & 78 & 35858 & 16335 & 16413 & 48 \\ 25 & 8 / 13 & 1072 & 88 & 37165 & 17642 & 17730 & 50 \\ 26 & 8 / 14 & 1084 & 100 & 38600 & 19077 & 19177 & 52 \\ 27 & 8 / 15 & 1087 & 103 & 39715 & 20192 & 20295 & 51 \\ 28 & 8 / 16 & 1098 & 114 & 40794 & 21271 & 21385 & 53 \\ 29 & 8 / 17 & 1114 & 130 & 41853 & 22330 & 22460 & 58 \\ 30 & 8 / 18 & 1127 & 143 & 43379 & 23856 & 23999 & 60\end{array}$


medRxiv preprint doi: https://doi.org/10.1101/2020.09.15.20195081; this version posted September 18, 2020. The copyright holder has placed this preprint (which was not certified by peer review) in the Public Domain. It is no longer restricted by copyright. Anyone can legally share, reuse, remix, or adapt this material for any purpose without crediting the original authors.

$\begin{array}{llllllll}31 & 8 / 19 & 1143 & 159 & 44771 & 25248 & 25407 & 63 \\ 32 & 8 / 20 & 1154 & 170 & 45938 & 26415 & 26585 & 64 \\ 33 & 8 / 21 & 1168 & 184 & 47045 & 27522 & 27706 & 66 \\ 34 & 8 / 22 & 1175 & 191 & 47946 & 28423 & 28614 & 68 \\ 35 & 8 / 23 & 1180 & 196 & 48716 & 29193 & 29389 & 67 \\ 36 & 8 / 24 & 1195 & 211 & 49794 & 30271 & 30482 & 69 \\ 37 & 8 / 25 & 1208 & 224 & 51034 & 31511 & 31735 & 70 \\ 38 & 8 / 26 & 1225 & 241 & 52169 & 32646 & 32887 & 73 \\ 39 & 8 / 27 & 1237 & 253 & 53318 & 33795 & 34048 & 74 \\ 40 & 8 / 28 & 1254 & 270 & 54650 & 35127 & 35397 & 76 \\ 41 & 8 / 29 & 1263 & 279 & 55471 & 35948 & 36277 & 77 \\ 42 & 8 / 30 & 1278 & 294 & 56109 & 36586 & 36880 & 80 \\ 43 & 8 / 31 & 1295 & 311 & 57130 & 37607 & 37918 & 82 \\ 44 & 9 / 1 & 1306 & 322 & 57735 & 38212 & 38534 & 84 \\ 45 & 9 / 2 & 1318 & 334 & 58827 & 39304 & 39638 & 84\end{array}$

$D_{12}$ : Accumulated number of deaths for $1^{\text {st }}+2^{\text {nd }}$ waves $D_{2}$ : Accumulated number of deaths for $2^{\text {nd }}$ wave

$r_{12}$ : Accumulated number of discharged for $1^{\text {st }}+2^{\text {nd }}$ waves $r_{2}$ : Accumulated number of discharged for $2^{\text {nd }}$ waves $R_{2}=D_{2}+r_{2}$ : Removed number for $2^{\text {nd }}$ wave $\lambda_{2}=D_{2} / R_{2}$ : Mortality rate for $2^{\text {nd }}$ wave $\left(\times 10^{-4}\right)$ 
medRxiv preprint doi: https://doi.org/10.1101/2020.09.15.20195081; this version posted September 18, 2020. The copyright holder has placed

this preprint (which was not certified by peer review) in the Public Domain. It is no longer restricted by copyright. Anyone can legally share,

reuse, remix, or adapt this material for any purpose without crediting the original authors.

\section{References}

[1] W. O. Kermack and A.G. McKendrick, "A Contribution to the Mathematical Theory of Epidemics", Proceedings of the Royal Society A115 , 700-721 (1927)..

[2] W. D. Murray, "Epidemic models and the dynamics of infectious diseases", Mathematical Biology 42, 610-650 (1993).

[3] H. Hethcote, "The Mathematics of Infectious Diseases", SIAM Review 42, 599-653 (2000).

[4] M.J. Keeling and L. Danon, "Mathematical modelling of infectious diseases ", British Medical Bulletin 92, 33-42 (2009).

[5] S. Pathak, A. Maiti and G.P. Samanta, "Rich dynamics of an SIR epidemic model", Nonlinear Analysis: Modelling and Control 15, 71-81 (2010).

[6] T. Harko, F.S.N. Lobo, M.K. Mak, "Exact analytical solutions of the SusceptibleInfected-Recovered (SIR) epidemic model and of the SIR model with equal death and birth rates", Applied Mathematics and Computation 236, 184194 (2014).

[7] J.C. Miller, "Mathematical models of SIR disease spread with combined non-sexual and sexual transmission routes", Infectious Disease Modelling 2, 3555 (2017).

[8] R. Sameni, "Mathematical Modeling of Epidemic Diseases; A Case Study of the COVID19 Coronavirus", [arXiv:2003.11371 [q-bio.PE]](2017).

[9] P-F. Verhulst, "Notice sur la loi que la population poursuit dans son accroissement", Correspondance Mathmatique et Physique 10, 113-121 (1838).

[10] T. Saito and K. Shigemoto, "A Logistic Curve in the SIR Model and Its Application to Deaths by COVID-19 in Japan ", [medRxiv 10.1101/2020.06.25.20139865] (2020).

[11] Data published from the Ministry of Health, Labor and Welfare of Japan. See for example, "Summary of the New Coronavirus Infection "(in Japanese), https://hazard.yahoo.co.jp/article/20200207; "Status of the Domestic New Coronavirus Infection"(in Japanese), https://toyokeizai.net/sp/visual/tko/covid19/. 